\title{
Choroidal thickness in both eyes of patients with unilateral central serous chorioretinopathy
}

\begin{abstract}
Background: Central serous chorioretinopathy (CSCR) afflicts the macula and is generally observed in males. The speculated pathophysiology is increased permeability in the choroidal vasculature. Optical coherence tomography (OCT) has enabled us to study the retina and choroid in unprecedented detail.
\end{abstract}

Purpose: The study employed swept-source optical coherence tomography (SS-OCT) to compare choroidal thickness in both eyes of unilateral CSR and compared it with age matched and gender matched normal subjects.

Methodology: Retrospective review was conducted in the diagnostics department of Amanat Eye Hospital on 40 patients with unilateral CSCR and age/gender matched 40 normal subjects. All patients underwent comprehensive SS-OCT examination. Choroidal thickness was measured from 9 different zones and values were averaged.

Results: The mean age in case and control group was $45.7 \pm 15.2$ years and $48.1 \pm 14.9$ years respectively. In affected, unaffected and normal eyes; subfoveal thickness was $335.4 \pm 87.7,337 \pm 87.7$ and $259 \pm 90.7 \mu \mathrm{m}$ respectively and averaged choroidal thickness (of the entire choroid) was $339.36 \pm 71.25,311.49 \pm 71.91$ and $241.99 \pm 82.53 \mu \mathrm{m}$ respectively. The subfoveal and averaged choroidal thickness between case and control group was significantly different with $\mathrm{p}<0.001$. While the difference of subfoveal and averaged choroidal thickness between affected and unaffected eye was insignificant with $\mathrm{p}=0.908$ and $\mathrm{p}=0.086$ respectively. The frequency of systemic hypertension between cases and controls was insignificant with $\mathrm{p}=0.431$.

Conclusion: The study revealed that the thickness of the choroid is increased not only in affected eye but also contralateral apparently unaffected eye of patients presenting with unilateral CSCR as compared to choroidal thickness of age matched normal eyes.

Keywords: central serous chorioretinopathy, choroidal thickness, swept source optical coherence tomography, indocyanine green angiography, choroidal vascular hyperpermeability
Volume 4 Issue 5 - 2016

\author{
Aamir Asrar,' Bisma Ikram, ${ }^{2}$ Hina Khan, ${ }^{2}$ \\ Maha Asrar ${ }^{3}$ \\ 'Department of Ophthalmology, Amanat Eye Hospital, Pakistan \\ ${ }^{2}$ Department of Ophthalmic Diagnostic, Amanat Eye Hospital, \\ Pakistan \\ ${ }^{3}$ Shifa College of Medicine, Pakistan
}

Correspondence: Hina Khan, Department of Ophthalmic Diagnostic, Amanat Eye Hospital, Pakistan, Tel 92322585 I I I3, Email drhina@amanathospital.com

Received: July 29, 2016 | Published: August 31, 2016
Abbreviations: CSCR, central serous chorioretinopathy; OCT, optical coherence tomography; SS, swept source; ICGA, indocyanine green angiography; RPE, retinal pigments epithelium

\section{Introduction}

Central serous chorioretinopathy (CSCR) is a detachment of the neurosensory retina involving the macula. It affects predominantly men in the most productive years of their lives. ${ }^{1}$ CSCR frequently involves one eye; however, both eyes are involved in $40 \%$ cases. $^{2}$ The exact etiology is unknown but conditions like Glucocorticoid levels, genetic predisposition, type A personality, pregnancy, cardiovascular diseases and hypertension, use of antibiotics, bone marrow or organ transplantation and infection of the respiratory tract identified as some of risk factors for CSCR. ${ }^{3-5}$ Most of them point towards a state of increased levels steroids in the body. i.e. hypercortisolism. Although only one eye is involved, it is speculated that increased cortisol level in the body increases the choroidal permeability. Therefore, theoretically, even though only one eye is affected choroidal changes should occur in both eyes.

The true pathophysiology of CSCR is not fully known. Published literatures ${ }^{6-9}$ presents different theories on the development of CSCR. Before the advent of Optical Coherence tomography (OCT), Fundus fluorescein angiography (FFA) was used to study CSCR and leakage pattern was defined. Changes observed on Indocyanine Green Angiography (ICGA) include delayed filling of choroidal arteries and chorio-capillaries, choroidal venous dilatation, and choroidal vascular hyperpermeability; and study ${ }^{10}$ suggest that choroidal abnormalities might be the origin of CSCR. OCT has enabled us to perform a more detailed non invasive structural analysis of the eye with axial resolution of 5-10microns. ${ }^{11-13}$ With newer machines using longer wavelength $(1050 \mathrm{~nm})$ as in the swept source OCT (SS-OCT), it is now possible to map the whole choroidal thickness in the entire extent of the macular area scan. It allows us to have new theories about CSCR. With the innovation of SS-OCT, high-acquisition speed, highresolution, enhanced-depth and improved tissue penetration) it is now possible to critically analyze layers of retina and choroid and provides new insights into choroidal and retinal disorders. Based on the above the current study aimed to determine the changes in choroidal thickness of the affected eyes as well as apparently normal eyes of patients with unilateral CSCR compared to age matched normal eyes.

\section{Methodology}

A retrospective study conducted in the settings of Amanat Eye Hospital. Consecutive sampling technique was used to collect the sample of 40 patients diagnosed with CSCR compared them with 40 age matched patients with normal retinas were enrolled in the study. 
All the patients, presenting with CSCR to Amanat Eye Hospital were included in the study. The study followed the tenets of Declaration of Helsinki. The internal review board of Amanat Eye Hospital approved the protocols of this study. Patients with unilaterally active CSCR were included in the study. Patients with bilateral CSCR, prior history of other vitreoretinal diseases or undergone any treatment for CSCR were excluded from the study, in order to eliminate the any potential influence on choroidal thickness.

All the subjects underwent a comprehensive ophthalmic examination on slit lamp biomicroscope by an Ophthalmologist. To visualize full choroidal thickness Swept Source OCT (Topcon Triton) was employed. SS OCT performs scanning with the speed of 50,000 scans per second with the beam of wavelength $1050 \mathrm{~nm}$. The choroid thickness is defined as the thickness from Bruch's membrane to the inner scleral border. Radial scans were taken and 3-Dchoroidal thickness maps were obtained. Choroidal thickness in 9 different zones were obtained namely; subfoveal central zone, nasal, temporal, superior and inferior of paracentral zones; and nasal, temporal, superior and inferior of peripheral zones (Figure 1). All the scans were performed by a trained ophthalmic technologist and analyzed by an experienced ophthalmologist.

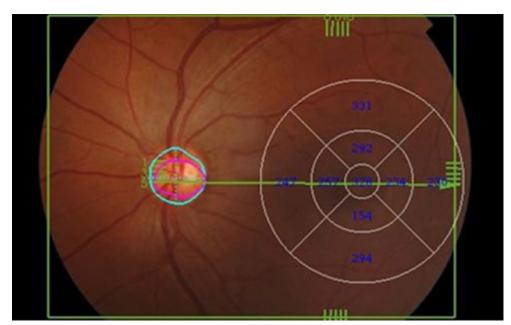

Figure I Grid: 9 different zones namely; subfoveal central zone, nasal, temporal, superior and inferior of paracentral zones; and nasal, temporal, superior and inferior of peripheral zones.

\section{Statistical analysis}

The Statistical Package for Social Sciences software (SPSS, version 22) was applied to organize and tabulate the data collected. Descriptive statistics of all variables were calculated and Pearson Chi square/ fisher exact test was applied to evaluate sex and systemic hypertension. Choroidal thickness of the eyes with CSCR and unaffected fellow eyes were compared with normal eyes by employing independent t-tests (two-tailed). T-test was also applied for the comparison of choroidal thickness of the eyes with CSCR and that of unaffected fellow eyes. The correlation between symptom duration and choroidal thickness was evaluated by Pearson's correlation. All the results were evaluated with confidence interval (CI) $95 \%$ and were considered significant at $p$ value $<0.05$. Bonferroni's correction was used for the adjustment of critical $\mathrm{p}$ values in order to avoid the possible inflation due to multiple comparisons.

\section{Results}

The mean age in the case group and control group was $45.7 \pm 15.2$ and $48.1 \pm 14.9$ years respectively. The male to female ratio was $36: 4$ and 30:10 in the patients with CSCR and patients with age matched a normal eye respectively; which is statistically insignificant $(\mathrm{p}=0.077)$. Out of $40,11(27.5 \%)$ patients with CSCR were having systemic hypertension and $8(20.0 \%)$ patients within control were having systemic hypertension; which is found to be insignificant $(\mathrm{p}=0.431)$ (Table 1). The subfoveal mean thickness was $335.4 \pm 87.7,337 \pm 87.7$ and $259 \pm 90.7 \mu \mathrm{m}$ in affected with CSR eye, unaffected eye and age matched normal eye respectively (Table 2). The subfoveal mean thickness was statistically significant in affected and unaffected eye of case group than control group with $\mathrm{p}<0.001$ but the mean difference between subfoveal thickness of affected and unaffected eye among case group was insignificant with $\mathrm{p}=0.908$.

Table I Demographic characteristics of normal individuals and patients with unilaterally active central serous chorioretinopathy

\begin{tabular}{|c|c|c|c|}
\hline & Case Group & Control Group & p Value \\
\hline No. of Patients & 40 & 40 & \\
\hline $\begin{array}{l}\text { Age (Years) } \\
\text { Mean } \pm S D\end{array}$ & $45.7 \pm 15.2$ & $48.1 \pm 14.9$ & \\
\hline $\begin{array}{l}\text { Sex Ratio } \\
\text { Male to Female }\end{array}$ & $36: 4$ & $30: 10$ & $\mathrm{P}=0.077$ \\
\hline $\begin{array}{l}\text { Systemic Hypertension } \\
\text { n (\%) }\end{array}$ & II (27.5\%) & 8 (20.0\%) & $\mathrm{p}=0.431$ \\
\hline
\end{tabular}

Table 2 Mean choroidal thickness in eyes with unilateral CSC, other unaffected fellow eyes and normal eyes

\begin{tabular}{|c|c|c|c|c|c|c|}
\hline & Affected Eye & Unaffected Eye & Normal Eye & P value $^{+}$ & P value ${ }^{++}$ & P value ${ }^{+++}$ \\
\hline $\begin{array}{l}\text { Subfoveal Thickness }(\mu \mathrm{m}) \\
\text { Mean } \pm \text { SD }\end{array}$ & $335.4 \pm 87.7$ & $337 \pm 87.7$ & $259 \pm 90.7$ & $p<0.001$ & $p<0.001$ & $p=0.908$ \\
\hline $\begin{array}{l}\text { Choroidal Thickness }(\mu \mathrm{m}) \\
\text { Mean } \pm \text { SD }\end{array}$ & $339.36 \pm 71.25$ & $311.49 \pm 71.91$ & $241.99 \pm 82.53$ & $\mathrm{P}<0.00 \mathrm{I}$ & $p<0.001$ & $\mathrm{P}=0.086$ \\
\hline
\end{tabular}

$\mathrm{P}$ value ${ }^{+}$Comparison between unilateral CSC eyes and normal eyes by t-test

$P$ value $^{++}$Comparison between unaffected fellow eyes and normal by t-test

$P$ value ${ }^{+++}$Comparison between unilateral CSC eyes and unaffected fellow eyes by t-test 
All the values of choroidal thickness taken from 9 different zones were averaged; the mean and standard deviation (SD) of averaged choroidal thickness in affected eyes, contralateral normal eyes of case group and age matched normal eyes of control group were $339.36 \pm 71.25, \quad 311.49 \pm 71.91$ and $241.99 \pm 82.53 \mu \mathrm{m}$ respectively. The mean value of averaged choroidal thickness of CSCR eyes were statistically significant than normal eye with $p<0.001$. Similarly the averaged choroidal thickness of apparently normal eye of case group were also significantly greater than the averaged choroidal thickness of control group with $p<0.001$. However, the difference of mean values of averaged choroidal thickness of CSCR affected eyes and contralateral normal eyes within case group was found to be insignificant with $\mathrm{p}=0.086$. The general linear model also illustrated similar results of averaged choroidal thickness differences between these three groups. Insignificant correlation of the averaged choroidal thickness of CSCR eye with symptom duration was found by employing pearson correlation with $\mathrm{r}=0.271, \mathrm{p}=0.227$ (Table 2 ).

\section{Discussion}

Blisters of fluid i.e. serous detachment underneath the macula, is the clinical hallmark of CSCR. It can be visualized clearly by the (SS-OCT) images. This study revealed that whatever the stage of the disease, the choroid was found to be thicker in affected CSCR eyes than the age matched normal eyes; similarly the unaffected eye of patients with CSCR, subfoveal and averaged choroidal thickness exceeded the normal value than similar age group (Figure 2). The results are comparable with the previous studies. The allegedly confounding factors i.e. hypertension and symptom duration were found to be independent with the choroidal thickness in the current study these results were also compatible with the previous literature.

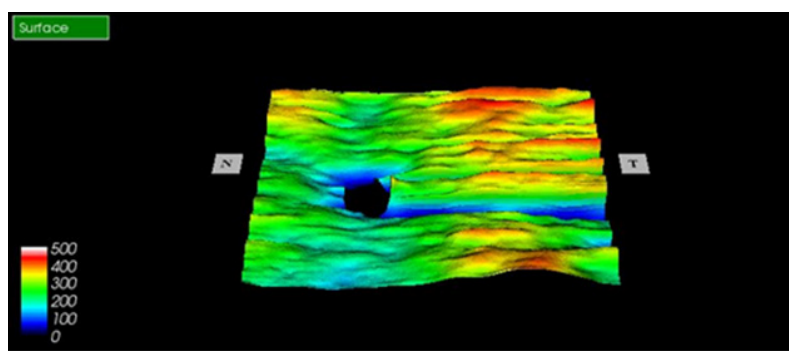

Figure 2 Age matched choroidal thickness colored map.

The abnormal increase in the thickness of choroid in apparently normal fellow eye of case group indicates that CSCR must essentially be a systemic disorder affecting both eyes at the choroidal level. ${ }^{14,15}$ In cross sectional studies ${ }^{16,17}$ the CSCR clinically and simultaneously involves both eyes with frequency ranging from 4 to $14 \%$ cases. However, in 20 to $30 \%$ cases bilaterality is reported when follow up was done for a long period of time. ${ }^{18}$ The study reported new leakages sites in normal eye of CSCR patients when follow up was done for 2 years. $^{7}$ Reports $^{6,19}$ on Indocyanine green angiography (ICGA) in CSCR revealed instability of choroidal circulation. These studies suggested that increase in choroidal vascular hyperpermeability might be the root cause of CSCR. A study ${ }^{7}$ in Japan reported 96\% patients of CSCR with increased choroidal vascular hyperpermeability. The choroid is believed to be stretched.

Study $^{20}$ using ICGA have concluded that choroidal vascular hyperpermeability was present in $62 \%$ of contralateral eyes of unilateral CSCR patients. Based on ICGA, another study ${ }^{6}$ suggested the rate was $65 \%$. These results indicate that the choroid might not always be abnormally thicker in the apparently normal fellow eyes of CSCR patients. However, in the current study choroid was thickened in all apparently normal eyes with unilateral CSCR which is an indirect validation of the proposed choroidal vascular hyperpermeability theory. So it may be incurred that choroidal vascular hyperpermeability was found in $100 \%$ in the current study, which is significantly higher than previously reported. The present study also took in to account CSCR patients diagnosed with SD OCT (EDI mode) at first but the measurements were generally lower than the SS OCT. Studies ${ }^{21,22}$ have shown that choroidal thickness measurements (especially when studying CSCR) when estimated manually with SD OCT are lower than the automated estimates provided with SS OCT. Therefore, while both these machines can detect abnormal choroidal thickness but cannot be used interchangeably.

The results of current study are compatible with previous studies and revealed that with the advent of SS-OCT; the retinal and choroidal layers can be assessed effortlessly with enhanced depth, high penetration ability of longer wavelength $1050 \mathrm{~nm}$. We are now able to measure choroidal thickness more precisely and subclinical choroidal abnormalities can easily be evaluated in patients with CSCR. The present study has employed investigations like the Indocyanine green angiography ICGA to measure the choroidal vascular hyperpermeability. Indeed a correlation between the structural parameter i.e. choroidal thickness and functional parameters (leakage on ICGA) is one direction in which this research can grow. Another avenue to extend this research is a longer follow up to gauge the pattern of choroidal thickness overtime.

\section{Conclusion}

The results of the studies indicates that the choroidal thickness in both eyes of patients with CSCR are abnormally higher than the age matched normal eyes indicating that it is part of systematic disorder. The pathology of CSCR entails that albeit it is present in one eye but effects can be seen in both eyes due to underlying systematic cause.

\section{Acknowledgments}

We would like to acknowledge the contribution of Ms Naila Boota, Mr. Mohammad Kashif, and Mr. Rizwan Waris for lending us their expertise in scan acquisition.

\section{Conflicts of interest}

The authors declare there are no conflicts of interest.

\section{Funding}

None.

\section{References}

1. Daruich A, Matet A, Dirani A, et al. Central serous chorioretinopathy: recent findings and new physiopathology hypothesis. Prog Retin Eye Res. 2015;48:82-118.

2. Spaide RF, Campeas L, Haas A, et al. Central serous chorioretinopathy in younger and older adults. Ophthalmology. 1996;103(12):2070-2079.

3. Nicholson B, Noble J, Forooghian F, et al. Central serous chorioretinopathy: update on pathophysiology and treatment. Surv Ophthalmol. 2013;58(2):103-126.

4. Errera MH, Kohly RP, Cruz L. Pregnancy associated retinal diseases and their management. Surv Ophthalmol. 2013;58(2):127-142. 
5. Eom Y, Oh J, Kim SW, et al. Systemic factors associated with central serous chorioretinopathy in Koreans. Korean $J$ Ophthalmol. 2012;26(4):260-264.

6. Maruko C, Iida T, Sugano Y, et al. Subfoveal choroidal thickness in fellow eyes of patients with central serous chorioretinopathy. Retina. 2011;31:1603-1608.

7. Iida T, Kishi S, Hagimura N, et al. Persistent and bilateral choroida vascular abnormalities in central serous chorioretinopathy. Retina. 1999;19(6):508-512.

8. Marmor MF. New hypotheses on the pathogenesis and treatmen of serous retinal detachment. Graefes Arch Clin Exp Ophthalmol. 1998;226(6):548-552.

9. Negi A, Marmor MF. Experimental serous retinal detachment and focal pigment epithelial damage. Arch Ophthalmol. 1984;102(3):445-449.

10. Spitznas M. Pathogenesis of central serous retinopathy: a new working hypothesis. Graefes Arch Clin Exp Ophthalmol. 1986;224(4):321-324.

11. Margolis R, Spaide RF. A pilot study of enhanced depth imaging optica coherence tomography of the choroid in normal eyes. Am J Ophthalmol. 2009;147(5):811-815.

12. Imamura Y, Fujiwara T, Margolis R, et al. Enhanced depth imaging optical coherence tomography of the choroid in central serous chorioretinopathy. Retina. 2009;29(10):1469-1473.

13. Spaide RF, Koizumi H, Pozzoni MC. Enhanced depth imaging spectral-domain optical coherence tomography. Am J Ophthalmol. 2008;146:496-500.

14. Haimovici R, Koh S, Gagnon DR, et al. Risk factors for central serous chorioretinopathy: a case-control study. Ophthalmology. 2004;111(2):244-249.
15. Tittl MK, Spaide RF, Wong D, et al. Systemic findings associated with central serous chorioretinopathy. Am J Ophthalmol. 1999;1128(1):63-68.

16. Lu JG, Friberg TR. Idiopathic central serous retinopathy in China: a report of 600 cases (624 eyes) treated by acupuncture. Ophthalmic Surg. 1987;18:608-611.

17. Bujarborua D, Chatterjee S, Choudhury A, et al. Fluorescein angiographic features of asymptomatic eyes in entral serous chorioretinopathy. Retina. 2005;25(4):422-429.

18. Kitzmann AS, Pulido JS, Diehl NN, et al. The incidence of central serous chorioretinopathy in Olmsted County, Minnesota, 1980-2002. Ophthalmology. 2008;115(1):169-173.

19. Kuroda S, Ikuno Y, Yasuno Y, et al. Choroidal Thickness in Central Serous Chorioretinopathy. Retinac. 2013;33(2):302-308.

20. Okushiba U, Takeda M. Study of choroidal vascular lesions in central serous chorioretinopathy using indocyanine green angiography. Nippon Ganka Gakkai Zasshi. 1997;101(1):74-82.

21. Yamashita T, Yamashita T, Shirasawa M, et al. Repeatability and reproducibility of subfoveal choroidal thickness in normal eyes of Japanese using different SD-OCT devices. Invest Ophthalmol Vis Sci. 2012;53(3):1102-1107.

22. Matsuo Y, Sakamoto T, Yamashita T, et al. Comparisons of Choroidal Thickness of Normal Eyes Obtained by Two Different Spectral-Domain OCT Instruments and One Swept-Source OCT Instrument. Invest Ophthalmol Vis Sci. 2013;54(12):7630-7636. 